\title{
Trapped magnetic field distribution above two magnetized bulk superconductors close to each other
}

\author{
M Houbart ${ }^{1}$, J-F Fagnard ${ }^{1}$, A R Dennis ${ }^{2}$, D K Namburi ${ }^{2}$, \\ Y Shi ${ }^{2}$, J H Durrell ${ }^{2}$ and $\mathbf{P}$ Vanderbemden ${ }^{1}$ \\ ${ }^{1}$ University of Liege, Department of Electrical Engineering and Computer \\ Science, B-4000 Liege, Belgium \\ ${ }^{2}$ University of Cambridge, Bulk Superconductivity Group, Cambridge CB2 \\ 1PZ, United Kingdom \\ E-mail: michel.houbart@uliege.be
}

\begin{abstract}
Bulk large-grain superconductors can be used as high-field permanent magnets. Although the properties of such individual trapped field magnets are well documented, much less is known concerning their behaviour when two are brought together. In this work, the interaction between two cylindrical bulk $\mathrm{YBa}_{2} \mathrm{Cu}_{3} \mathrm{O}_{7}$ (YBCO) superconductors is described. Two sets of experiments were carried out. The first involved the simultaneous magnetization of two bulk superconductors placed a short distance apart. Here, the applied magnetic field was aligned parallel to the $c$-axis of one bulk, while the other was oriented with its $c$-axis offset. For a centre-to-centre distance equal to twice the sample height, the presence of the second sample is found not to alter the current distribution inside the first. Consequently, the contribution of both samples simply sums, thus increasing the magnetic flux density between them. In the second set of experiments, the translational approach of the superconductors with parallel $c$-axes was investigated. The following configurations were considered: (i) face to face approach (with anti-parallel trapped field orientation) and (ii) sideways approach (with parallel trapped field orientation). An irreversible decrease of the trapped field was measured on separation . Repeated approach cycles showed that the irreversible loss of trapped field is largest for the first approach.
\end{abstract}

Bulk superconductor, trapped field magnet, magnetic field gradient, interacting bulk superconductors, flux pinning 


\section{Introduction}

The ability of bulk superconductorsto trap large magnetic fields makes them attractive for a broad range of applications [1-5]. In particular, a magnetized bulk superconductor can act as a pseudo-permanent or 'trapped field' magnet. Unlike conventional permanent magnets, which are limited by their saturation magnetization, e.g. 1.4 tesla for Nd-Fe-B [6], superconductors do not exhibit the same limitation. Their trapped field ability can be enhanced by increasing their dimensions [7]. The maximum field trapped in a bulk super-

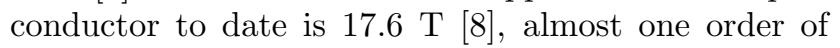
magnitude higher than the fundamental limit for iron. The primary limiting factor impeding the realization of higher trapped fields is the mechanical robustness required to withstand the Lorentz force [8-11].

Superconducting trapped field magnets can be used to produce both a large flux density $\mathbf{B}$ and a large gradient of flux density $\nabla \mathbf{B}$. One example where a large $\mathbf{B}$ is desirable is in electric synchronous motors to increase the system power density $[1,5,12,13]$. Large gradients are required in levitation systems [12], magnetic bearings [12] and magnetic drug delivery $[1,14-18]$.

In this context, combining several similar bulk superconductors is of interest in order to increase the flux density and modify the flux density distribution [19], in a similar way as when permanent magnets are combined in a Halbach array [20]. This is however less straightforward when combining superconducting trapped field magnets, for two reasons. The first is that the superconductors need to be magnetized. It is therefore relevant to understand how two superconductors behave when they are magnetized simultaneously. The second reason is related to the fact that the field generated by a superconductor arises from persistent macroscopic current loops. The distribution and the amplitude of the trapped current loops of one sample could be altered by the proximity of another neighbouring sample, leading to a demagnetization. The situation is actually comparable to a "crossed-field" experiment $[21,22]$ with the difference being that the field component perpendicular to the magnetization is not generated by a stable field source but by a neighbouring superconductor.

The crossed-field effect has been investigated ex- perimentally, analytically and numerically by considering a pre-magnetized bulk superconductor subjected to $N$ successive cycles of transverse field [23-29]. In this case, the decay of the magnetization can be fitted using a power law $M \sim N^{-a}$, where $a$ is an increasing function of the transverse field amplitude. In addition, finite element simulations suggest that the decay of the magnetization resulting from the first few cycles is associated with a redistribution of the superconducting current inside the bulk superconductor, while the decay resulting from further cycles is related to a decrease of the superconducting current density [23]. These results underline that superconducting trapped field magnets are sensitive to an external magnetic field that is not parallel to the main axis of their magnetization, a situation that is inevitable when two magnetized bulk superconductors are placed next to each other.

In this work, two different configurations of two interacting $\mathrm{YBa}_{2} \mathrm{Cu}_{3} \mathrm{O}_{7}$ (YBCO) bulk superconductors were investigated, as shown in figure 1 . In the first two nominally identical bulk superconductors were placed a short distance apart and magnetized simultaneously. The applied magnetic field was parallel to the $c$-axis of one of them, while the other had its $c$-axis misoriented at different angles. In the second configuration we investigated how the trapped field distribution is affected when two permanently magnetized bulk superconductors are approached with parallel $c$-axes, either face to face (with anti-parallel trapped field orientation) or sideways (with parallel trapped field orientation).

The potentially detrimental effect of using an offaxis magnetization field has been studied experimentally for a single bulk sample [30,31]. It was reported that field cooled GdBCO bulk cryomagnets were able to almost maintain their full trapped field potential along their $c$-axis for magnetization field inclination up to $30^{\circ}$. This obervation might be attributed to the anisotropy of critical current density $(a-b$ plane vs. $c$-axis), which tends to align the flux lines with the c-axis even in off-axis magnetization. There are very few reports involving several trapped field superconductors placed together. Two staggered rows of stationary samples with parallel magnetizations have been used for generating a DC field varying sinusoidally in space [32-34]. Combining flux trapping and flux exclusion with samples having different critical temperatures or operating temperatures have been shown 
(a) Simultaneous magnetization

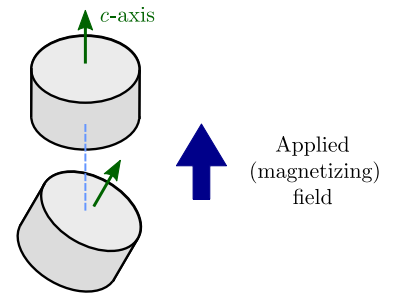

(b) Translational approach of pre-magnetized samples
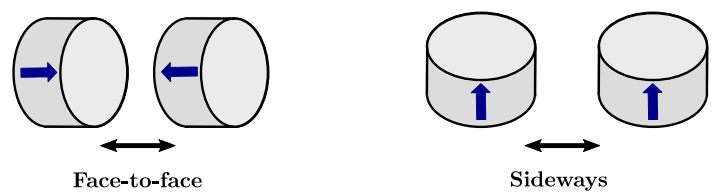

Figure 1. Schematic of the experiments carried out for investigating (a) simultaneous magnetization and (b) postmagnetization approach.

to yield magnetic field concentration effects ('magnetic lens') $[35,36]$. A circular Halbach array of superconductors for rotating machines was proposed by Hull et al [37] but the numerical optimization of the field generated by such an array was carried out only recently [38], using superconducting coils. An understanding of how a pair of neighbouring trapped field superconducting magnets behave in rather simple configurations is, therefore, still lacking.

\section{Experimental methods}

Experiments were performed to study the interactions between two (nominally identical) cylindrical $\mathrm{YBa}_{2} \mathrm{Cu}_{3} \mathrm{O}_{7}$ bulk superconductors. The samples were fabricated by the TSMG method [39-41]. A preliminary Hall probe mapping of the flux density above the top surface of each sample was performed. The samples were magnetized through a field cooling process, which involved applying a magnetic field of $1.3 \mathrm{~T}$, cooling the sample to $77 \mathrm{~K}$ using liquid nitrogen and then removing the magnetic field. The resulting trapped field was measured using an array of 18 rotating Hall probes with a distance of about $3.5 \mathrm{~mm}$ between each probe. The distance between the probes and the surface of the sample was approximately $0.7 \mathrm{~mm}$. The exact dimensions and the maximum value of the trapped field on the surface of the samples are listed in table 1 .

Table 1. Dimensions and trapped field value at $77 \mathrm{~K}$ for the two investigated superconductors.

\begin{tabular}{lccc}
\hline $\begin{array}{l}\text { Sample } \\
\text { number }\end{array}$ & $\begin{array}{c}\text { Diameter } \\
{[\mathrm{mm}]}\end{array}$ & $\begin{array}{c}\text { Height } \\
{[\mathrm{mm}]}\end{array}$ & $\begin{array}{c}\text { Trapped field on } \\
\text { the surface }[\mathrm{T}]\end{array}$ \\
\hline 1 & 15.79 & 8.75 & 0.51 \\
2 & 15.61 & 8.84 & 0.52 \\
\hline
\end{tabular}

The experimental set up and procedure used for each interaction are presented in the following subsections.

\subsection{Simultaneously magnetized superconductors}

The magnetization process of an isolated superconductor was investigated in a first set of experiments as a reference case. The sample was always magnetized at $65 \mathrm{~K}$ both in field cooling under $2.5 \mathrm{~T}$ and in zero field cooling under $7 \mathrm{~T}$. The external field was applied with an angle, $\alpha$, with respect to the $c$-axis of the sample. A flux creep period of $20 \mathrm{~min}$ was allowed before heating the system up to $77 \mathrm{~K}$. The ramp-up and the ramp-down of the external field were performed at a constant rate of $1 \mathrm{mT} \cdot \mathrm{s}^{-1}$ and the heating up of the system was also carried out at a constant rate equal to $0.2 \mathrm{~K} \cdot \mathrm{min}^{-1}$. The magnetic flux density was recorded during the whole process at five locations on the surface of the superconductor at a sampling rate of $1 \mathrm{~Hz}$, as shown schematically in figure 2. The experimental data at $77 \mathrm{~K}$ presented in section 4 thus corresponds to the remaining trapped field after having heated the sample from $65 \mathrm{~K}$ to $77 \mathrm{~K}$. These measurements were carried out using Hall probes (LAKESHORE HGT-2101) in a bespoke mounting which was glued on the surface of the sample.

The influence of a second superconductor was then investigated by performing the same magnetization process in the presence of an additional superconducting sample with a $c$-axis aligned with the applied field. In this second series of experiments, the distance between the centres of the superconductors was approximately equal to $16 \mathrm{~mm}$ (which almost corresponds to their average diameter, i.e. $15.7 \mathrm{~mm}$ ) and the magnetic flux density was measured on the surface of the variable angle sample.

Both sets of experiments were carried out using a sample holder designed to allow the mounting of either one or two cylindrical samples inside a 12 T cryogenfree superconducting magnet. The chosen material for the sample holder was copper both for its small magnetic susceptibility $\left(-8.16 \cdot 10^{-8}\right.$ at $\left.77 \mathrm{~K}[42]\right)$ and its large thermal conductivity $\left(400-550 \mathrm{~W} \cdot \mathrm{m}^{-1} \mathrm{~K}^{-1}\right.$ in the range [77-300] $\mathrm{K}[43])$.

Figure 2 illustrates the experimental principle, as well as the position of the Hall sensors. The distance between two neighbouring sensors was $(3.2 \pm 0.2) \mathrm{mm}$. Figure 3 shows two views of the actual experimental system. Table 2 summarizes all the experiments carried out in this configuration. 


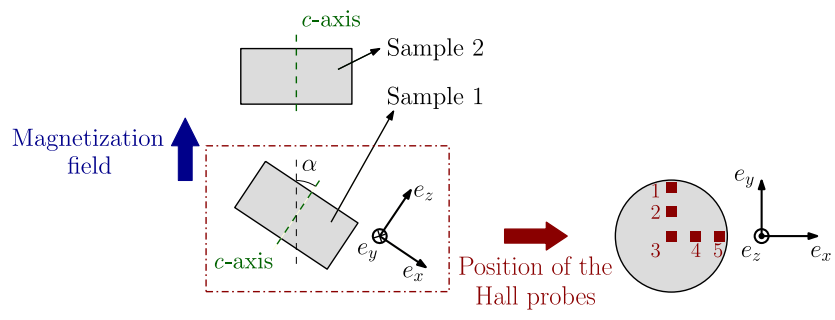

Figure 2. Schematic representation of the experiment on simultaneously magnetized superconductors. The magnetic flux density is measured on the surface of the sample 1 . The sample 2 is present only for experiments involving two superconductors.

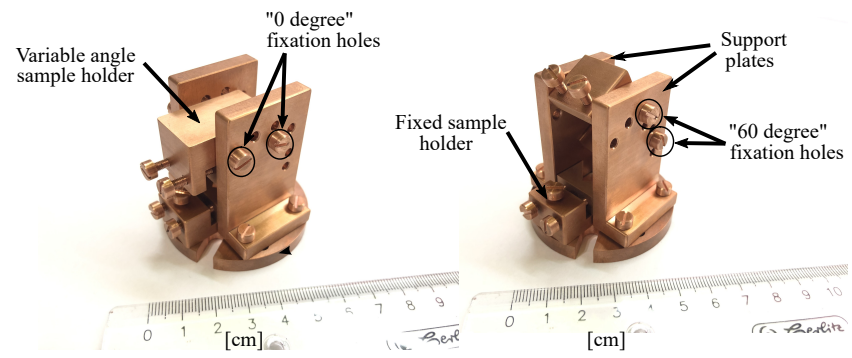

Figure 3. Views of the actual experimental system used for simultaneously magnetized superconductors experiment in two different configurations. On the left, the samples have their symmetry axis aligned with one another. On the right, the symmetry axes of the samples form an angle of $60^{\circ}$.

Table 2. Listing and codification of the experiments performed on the simultaneously magnetized superconductors.

\begin{tabular}{lccc}
\hline$\alpha$ & Number of samples & Magnetization & Codification \\
\hline $0^{\circ}$ & 1 & Field cooling & $0 / 1 / F C$ \\
$30^{\circ}$ & 1 & Field cooling & $30 / 1 / F C$ \\
$30^{\circ}$ & 1 & Zero field cooling & $30 / 1 / Z F C$ \\
$0^{\circ}$ & 2 & Field cooling & $0 / 2 / F C$ \\
$30^{\circ}$ & 2 & Field cooling & $30 / 2 / F C$ \\
$30^{\circ}$ & 2 & Zero field cooling & $30 / 2 / Z F C$ \\
$45^{\circ}$ & 2 & Field cooling & $45 / 2 / F C$ \\
\hline
\end{tabular}

\subsection{Post magnetization approach}

For this second type of interaction, both face to face (with anti-parallel magnetization) and sideways (with parallel magnetization) relative motions were investigated. The samples were first magnetized one after the other at $77 \mathrm{~K}$ (in a liquid nitrogen bath) using a field cooling procedure from $1.2 \mathrm{~T}$. During this magnetization process, the applied field was parallel to the $c$-axis of the superconductor and removed at a constant rate of $1 \mathrm{mT} \cdot \mathrm{s}^{-1}$. The magnetic flux density was sampled at a rate of $1 \mathrm{~Hz}$ at five locations on the surface of the first magnetized sample. After the magnetization of the second sample, a period of 20 min was allowed for flux creep to occur. The samples were then transferred into a mechanical system allowing translational motion while keeping the temperature at $77 \mathrm{~K}$. This system was made up of two carriages. The first was fixed to the external frame and the second was connected to a miniature linear actuator (AcTuOnix L16-P). This movable carriage could be displaced over a range of 140 $\mathrm{mm}$ at a maximum speed of $8 \mathrm{~mm} \cdot \mathrm{s}^{-1}$. Each carriage was equipped with a "sample holder base" allowing the accommodation of a sample holder in two positions (one for each type of approach). These sample holder bases were made of a thermally insulating material (G10) and were partly immersed in liquid nitrogen. The temperature of the superconducting samples could thus be kept at $77 \mathrm{~K}$ while maintaining the top part of the system at ambient temperature.

Five "approach/retract" cycles were performed in the face to face configuration. One cycle consisted in decreasing the distance $d$ between the samples from $81.6 \mathrm{~mm}$ to $21.6 \mathrm{~mm}$ in steps of $5 \mathrm{~mm}$; then decreasing it further to $4.4 \mathrm{~mm}$ in steps of $2.5 \mathrm{~mm}$ and finally going back to the initial distance by going through the same steps. The magnetic flux density was measured at each step at five locations on the surface of the stationary sample, as shown schematically in figure 4. A similar approach procedure was used for the sideways configuration, the only difference being that the distance between the samples ranges from $77.2 \mathrm{~mm}$ to 0 , i.e. contact between them.

Figure 4 illustrates the experiment principle as well as the Hall probes positions. The distance between two neighbouring sensors was $(3.2 \pm 0.2) \mathrm{mm}$. Figure 5 shows a schematic representation of the experimental system.

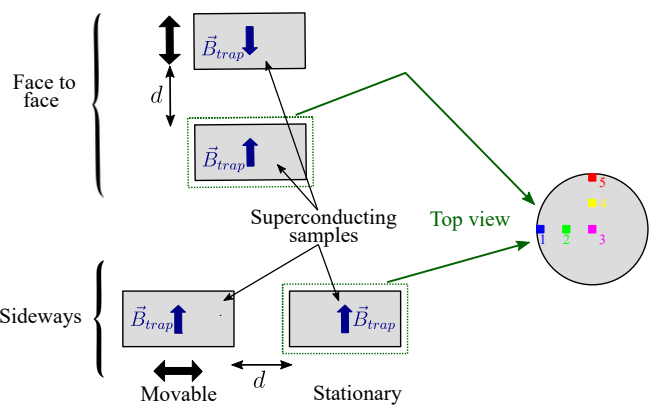

Figure 4. Schematic representation of the principle of the post magnetization approach experiment. The positioning of the Hall probes is represented on the top view of the stationary sample.

For comparison, the same "approach/retract" cycles were also performed and measured at ambient temperature with cylindrical $\mathrm{Nd}-\mathrm{Fe}-\mathrm{B}$ permanent magnets (diameter $15 \mathrm{~mm}$, height $8 \mathrm{~mm}$ ). Table 3 summarizes the experiments carried out. 


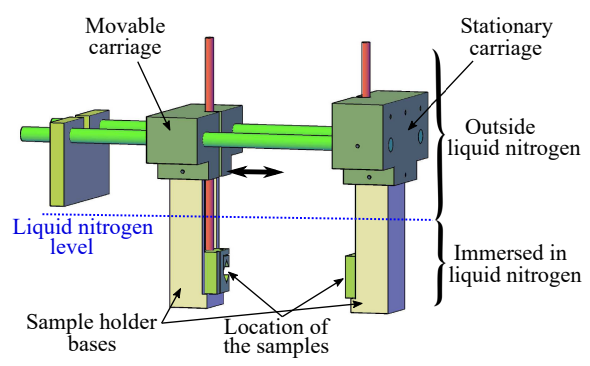

Figure 5. Schematic representation of the experimental system used for the post magnetization approach experiment.

Table 3. Numbering of the experiments carried out in the framework of the post magnetization approach.

\begin{tabular}{lll}
\hline Experiment number & Approach & Samples \\
\hline 1 & Face to face & Permanent magnets \\
2 & Sideways & Permanent magnets \\
3 & Face to face & Bulk superconductors \\
4 & Sideways & Bulk superconductors \\
\hline
\end{tabular}

\section{Analytical model}

A simple analytical model of the field generated by a magnetized cylindrical bulk superconductor was developed. This model is based on analytical expressions of the magnetic flux density of a coil of finite height $[44,45]$. Assuming a completely magnetized superconductor in the sense of the Bean model [46] with current loops strictly parallel to the $a-b$ planes, an analytical expression can be found for the trapped field. This expression corresponds to the integration of the formula in [44] over the radius of the superconductor. For a superconductor of height $L_{s}$, radius $a_{s}$ and critical current density $J_{c}$, the radial and axial components of the trapped field can respectively be written as:

$$
\begin{aligned}
& B_{r}=\int_{0}^{a_{s}} \frac{\mu J_{c}}{\pi} \sqrt{\frac{\tilde{a}}{r}}\left[\frac{2-\tilde{k}^{2}}{2 \tilde{k}} K(\tilde{k})-\frac{E(\tilde{k})}{\tilde{k}}\right]_{\xi_{-}}^{\xi_{+}} \mathrm{d} \tilde{a}, \\
& B_{z}=\int_{0}^{a_{s}} \frac{\mu J_{c}}{4}\left[\frac{\xi \tilde{k}}{\pi \sqrt{\tilde{a} r}} K(\tilde{k})+\frac{(\tilde{a}-r) \xi}{|(\tilde{a}-r) \xi|} \lambda_{0}(\tilde{\varphi}, \tilde{k})\right]_{\xi_{-}}^{\xi_{+}} \mathrm{d} \tilde{a},(2)
\end{aligned}
$$

where $r$ and $z$ are respectively the radial and axial coordinates from the centre of the superconductor, $K$ and $E$ are complete elliptic integrals of the first and second kind, $\lambda_{0}$ is the Heuman Lambda function, $\xi_{ \pm}=$ $z \pm L_{s} / 2$ and $\tilde{k}$ and $\tilde{\varphi}$ are defined as:

$$
\begin{aligned}
\tilde{k}^{2} & =\frac{4 \tilde{a} r}{\xi^{2}+(\tilde{a}+r)^{2}}, \\
\tilde{\varphi} & =\tan ^{-1}\left|\frac{\xi}{\tilde{a}-r}\right| .
\end{aligned}
$$

This analytical model was used to determine the theoretical magnetic flux density sensed by the 5 Hall probes taking into account their location and orientation (see figures 2 and 4). The Hall probes are assumed to lie in a plane perfectly parallel to the sample surface. In the configurations involving two samples with or without parallel axes, the formulas above can be used to estimate analytically the resulting $\vec{B}$ at the Hall probe locations. This estimate assumes a simple vector summation of the flux density generated by each superconductor. It should be highlighted that this model assumes that current loops are strictly parallel to the $a-b$ planes even for samples magetized in an off-axis configuration. This simplification consists in neglecting the $c$-axis component of the critical current density. While open to challenge, this approach is reasonable given that the critical current density along the $c$-axis is experimentally found to be much smaller than that along the $a-b$ planes [47-49].

The model was fitted to the measurements obtained through a preliminary Hall probes mapping at $77 \mathrm{~K}$ to determine the critical current density of each sample $\left(1.6 \cdot 10^{4} \mathrm{Acm}^{-2}\right.$ and $1.5 \cdot 10^{4} \mathrm{Acm}^{-2}$ both assumed to be magnetic field-independent). A second mapping was also performed after having carried out the two sets of experiments. The fitting of the analytical model to this second mapping lead to the same critical current densities values. Therefore, the two sets of experiments did not enhance or degrade the critical current density of the superconductors. Using these critical current densities, the model was fitted to the results of the experiment $0 / 1 / F C$ at $77 \mathrm{~K}$ to determine the distance between the Hall probes plane and the surface of the measured sample $(0.4 \mathrm{~mm})$. From the $J_{c}$ values at $77 \mathrm{~K}$ and the trapped field measurements of the central sensor at $65 \mathrm{~K}$ and $77 \mathrm{~K}$, the critical current density of each sample at $65 \mathrm{~K}$ was also computed $\left(3.2 \cdot 10^{4} \mathrm{Acm}^{-2}\right.$ and $\left.3 \cdot 10^{4} \mathrm{Acm}^{-2}\right)$. This calculation was performed by assuming that the trapped field at the centre of the sample surface is given by the critical current density multiplied by a geometrical constant independant of temperature (see (1) and (2)).

The same model was extended to the case where the sample is not completely magnetized (see section 4.1.2 below). In this case, it is assumed that the current density $J_{c}$ flows azimuthally in the sample, except in a cylindrical core located at the centre of the sample. The determination of the height $h$ and radius $a$ of this current-free zone is also exposed in section 4.1.2. In all cases the critical current density is assumed to be field-independent. 


\section{Results and discussion}

\subsection{Simultaneously magnetized superconductors}

As discussed in the experiment description, trapped field measurements were performed between $65 \mathrm{~K}$ and $77 \mathrm{~K}$. Here we focus more particularly on the two extreme temperatures which correspond respectively to liquid nitrogen boiling temperature at $\sim 10^{-2}$ mbar and at ambient pressure.

4.1.1. Flux creep analysis. The time-dependence of the trapped flux density measured at the centre of the sample top surface was analysed in the framework of the flux creep model introduced by Zeldov [50,51]. The model assumes a constant temperature and a $J_{c}$ independent of $B$. According to extensions of this model, the trapped field at the end of the magnetization process should evolve following a power law of the form [52]:

$B(t)=B_{0}\left(1+\frac{t}{t_{0}}\right)^{\frac{1}{1-n}}$,

where the origin of time corresponds to the moment when the applied field hits $0 \mathrm{~T}$ during the magnetization process.

For each experiment, this equation was fitted to the flux creep measurements to approximate the value of the critical exponent $(n)$ at the location of each Hall sensor. The results are summarized in table 4 .

Table 4. Critical exponent values obtained from the fit of (5) on the measurements of the simultaneously magnetized superconductors experiments at $65 \mathrm{~K}$. For experiment $0 / 1 / F C$, a problem occurred during the data acquisition of the first Hall probe so no fit could be performed.

\begin{tabular}{llllll}
\hline Experiment & $n_{1}$ & $n_{2}$ & $n_{3}$ & $n_{4}$ & $n_{5}$ \\
\hline $0 / 1 / F C$ & $/$ & 39 & 36 & 36 & 40 \\
$30 / 1 / F C$ & $\mathbf{5 4}$ & $\mathbf{4 7}$ & $\mathbf{4 9}$ & 37 & $\mathbf{3 2}$ \\
$30 / 1 / Z F C$ & $\mathbf{5 5}$ & $\mathbf{4 5}$ & 40 & 37 & 36 \\
$0 / 2 / F C$ & 36 & 37 & 36 & 36 & 38 \\
$30 / 2 / F C$ & $\mathbf{5 2}$ & $\mathbf{4 7}$ & $\mathbf{4 8}$ & 36 & $\mathbf{3 1}$ \\
$30 / 2 / Z F C$ & $\mathbf{5 4}$ & $\mathbf{4 4}$ & 39 & 36 & $\mathbf{3 5}$ \\
$45 / 2 / F C$ & $\mathbf{8 2}$ & $\mathbf{7 4}$ & $\mathbf{9 9}$ & $\mathbf{5 4}$ & 36 \\
\hline
\end{tabular}

Considering the experiment $0 / 1 / F C$ as a reference case, it appears from table 4 that the value of the critical exponent over the surface of the sample when it is in the critical state lies in between 36 and 40. This is in agreement with critical exponents measured on melt-textured YBCO at the same temperature [53]. This observation remains true for the experiment $0 / 2 / F C$. As soon as values of $\alpha$ other than $0^{\circ}$ are involved, however, a modification of the critical exponent is observed for some sensors, highlighted in bold red font in table 4 . This modification highlights a change in the trapped field gradient at the location of the concerned sensors when compared to the reference case. This change in trapped field gradient can potentially result from the partial magnetization of a superconductor [54], which will be further discussed later.

4.1.2. Trapped field profile at $65 \mathrm{~K}$. The measurements of the field cooled experiments at $65 \mathrm{~K}$ are represented by the symbols in figures 6 and 7 . These measurements were performed on the surface of the bulk with varying orientation along two directions $\vec{e}_{x}$ and $\vec{e}_{y}$, where $\vec{e}_{y}$ is parallel to the axis of rotation of the variable angle sample. The classic conical shape is obtained for the magnetic flux density profile. The central trapped field value is, however, smaller and smaller for increasing value of $\alpha$.

The results plotted in figures 6 and 7 were further analyzed through a confrontation to analytical predictions. The superconductors were assumed to be in the critical state and their individual contributions to the magnetic flux density at the location of the sensors were computed using (1) and (2) with the critical current densities determined in section 3. The predictions of the model was then compared to the measurements and two distinct cases could potentially be observed:

- If a good agreement is obtained, it means that the samples are indeed in the critical state. The current distribution inside each of them is not altered by the presence of the other during the magnetization.

- If the measured trapped field is smaller than the modelled value, it means that the current distribution in at least one of the sample is not the assumed one. An alternative current distribution is therefore required to obtain a good agreement with experimental data.

This comparison for the experiments involving one sample is presented in figure 6 .

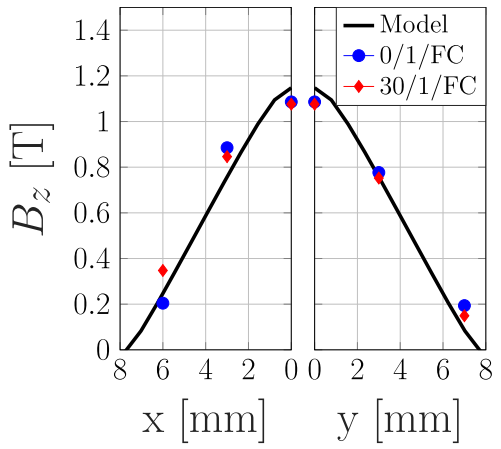

Figure 6. Comparison between the measured trapped field profile in field cooled experiments involving one sample at 65 $\mathrm{K}$ (symbols) and the trapped field profile computed using the model presented in section 3 (plain lines).

As can be observed in figure 6 , the misaligned sample is able to maintain almost its full trapped field potential in experiment $30 / 1 / F C$, which is in good agreement with the observations in $[30,31]$. The agreement with analytical predictions is also very satisfying. The current distribution 
is therefore likely to be the assumed one.

The comparison between the analytical predictions and the experiments results involving two samples are presented in figure $7(\mathrm{a}),(\mathrm{b})$ and $(\mathrm{c})$.

For the experiment $0 / 2 / F C$, the agreement of the measured data points with the analytical predictions (plain line in figure $7(\mathrm{a})$ ) is satisfying. This suggests that despite the close proximity of the samples, the current distribution in each of them is not altered by the presence of the other one.

This agreement, however, is no longer achieved for the central Hall sensor in experiments $30 / 2 / F C$ and $45 / 2 / F C$. In this case, the measured trapped field is smaller than expected. A potential explanation could consist in the partial magnetization of the misaligned superconductor. This possibility is suggested by considering the flux density at the centre of the bulk superconductor in the critical state: $B_{p}=2.49[\mathrm{~T}]$ (computed analytically using equation (2)). The field $B_{p}$ corresponds, to a first approximation, to the full penetration field of the sample. This field can be compared to the initial magnetization field component parallel to the $c$-axis of the misaligned sample: $B_{c-a x i s}=$ $2.5 \cdot \cos (\alpha)$ T. Assuming that only $B_{c-a x i s}$ contributes to the magnetization of the misaligned superconductor, it appears that the applied field would not be large enough to fully magnetize the sample in the experiments $30 / 2 / F C$ and $45 / 2 / F C$. To recover a good agreement with experimental data, a simple model of the trapped field generated by a partially magnetized superconductor was elaborated. This model assumes the presence of a cylindrical core (radius $a$, height $h$ ) free of current at the centre of the sample. The associated trapped field is thus computed by subtracting the contribution of this core in (1) and (2). A simplifying assumption is made in order to estimate the radius of the central core. At first order, we assume that this radius is the same as that of an inifinitely long superconducting cylinder. This leads to the following relation [55]:

$a=a_{s}\left(1-\frac{B_{c-a x i s}}{B_{p}}\right)$,

where $a_{s}$ is the radius of the bulk superconductor. The height of the core is kept as a free parameter to fit the model to the experimental data. The computed value of $h$ for experiments $30 / 2 / F C$ and $45 / 2 / F C$ are respectively given by $7.1 \mathrm{~mm}$ and $8 \mathrm{~mm}$. This simplified method for estimating the dimensions of the unsaturated core is useful given that the computation of the exact current density distribution in a partially magnetized cylindrical bulk superconductor is complex and requires numerical modelling. For our approximate estimation, the values found for $a$ and $h$ are in fair agreement with numerical simulations performed on cylindrical superconductors of similar aspect ratio $[55,56]$.

The predictions of the modified model are plotted against the experimental measurements for $\alpha=30^{\circ}$ and $45^{\circ}$ in figures $7(\mathrm{~d})$ and (e). A much better agreement is obtained with the modified model. The incomplete magnetization results in a flattening of the trapped field profile and can also explain the high value of the critical exponent observed in the analysis of the flux creep. In spite of the incomplete magnetization process, the experimental results plotted in figure 7 are in agreement with the picture in which the flux density distribution above the tilted sample simply results from the a simple vectorial addition of flux densities generated by the persistent currents in both samples.

A further possible cause for the disagreement in figures 7 (b) and (c) could be due to in the current loops being not strictly parallel to the $a-b$ planes, as assumed in the analytical model. This would result in a shifting of the overall magnetization of the misaligned sample towards the applied field direction. The magnetic flux density component measured by the sensors would therefore be smaller. A complete 3D modeling of the misaligned configurations could prove very helpful for determining the actual current density distributions.

4.1.3. Trapped field profile at $77 \mathrm{~K}$. A similar comparison between the experimental results and the analytical predictions was carried out with the measurements at $77 \mathrm{~K}$. The same conclusion as in the previous section can be drawn from the results of experiments $0 / 1 / F C$ and $30 / 1 / F C$ at $77 \mathrm{~K}$. The comparison to analytical predictions for these experiments is thus not presented here for conciseness. The results of the experiments involving two samples, however, are presented in figure 8 . The predictions of the model assuming completely magnetized samples are also shown.

In this temperature regime, the mismatching between measurements and analytical predictions at the location of the central sensor is no longer observed in the experiments $30 / 2 / F C$ and $45 / 2 / F C$ (figure 8 (b) and (c)). Therefore, the current distribution in each superconductor seems to be unaltered by the presence of the other, even in the misaligned cases. This would mean that starting from a different current density distribution at $65 \mathrm{~K}$, the misaligned sample reached the critical state by being heated up to $77 \mathrm{~K}$. The measured flux density is in agreement with a vectorial addition of the $\mathbf{B}$ vectors generated by each sample both assumed to be fully magnetized, which suggests two things. Firstly, an almost full magnetization can be reached, even for the sample cooled in an off-axis field. Secondly, given the centre-to-centre distance (of the order of the sample diameter), the two samples are not located close enough to be influenced significantly by one another.

4.1.4. Central value of the trapped field The magnetic flux density measured at the center of the superconductor surface $\left(B_{c}\right)$ at the end of the flux creep period (at $65 \mathrm{~K}$ ) as well as after having heated the system up to $77 \mathrm{~K}$ are presented in table 5 .

At $65 \mathrm{~K}$, when comparing the central trapped field of an isolated sample (measured in $0 / 1 / F C$ ) to the results of the experiments involving two samples, it appears that the second sample has a beneficial impact on the trapped field when $\alpha \leq 30^{\circ}$. This is no longer the case for the experi- 


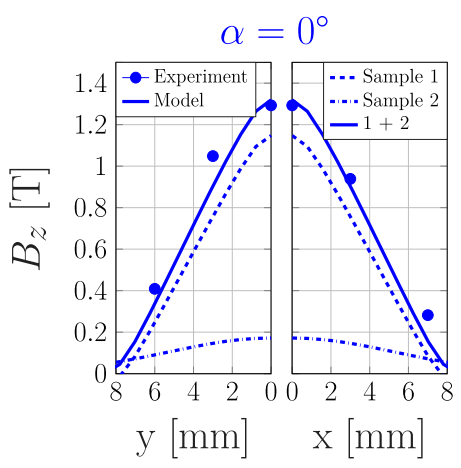

(a)

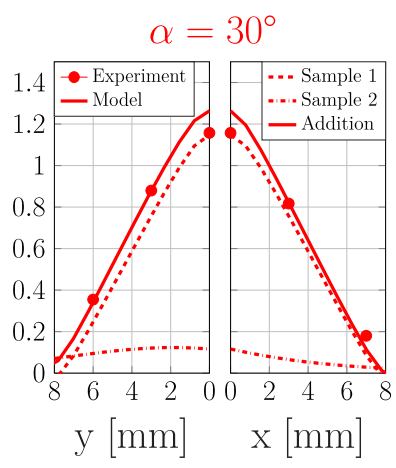

(b)

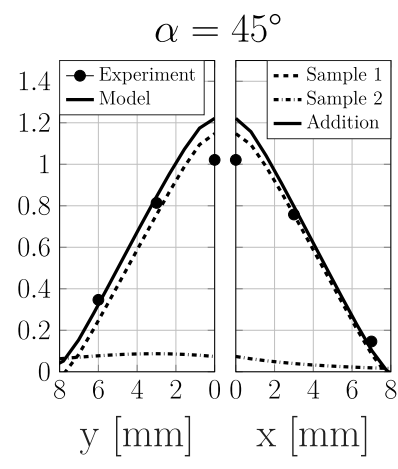

(c)

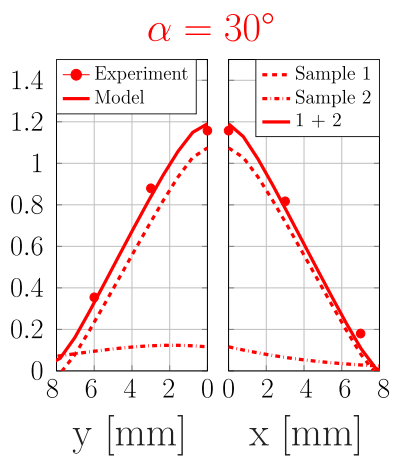

(d)

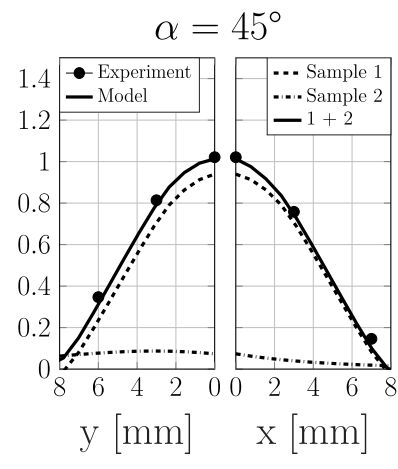

(e)

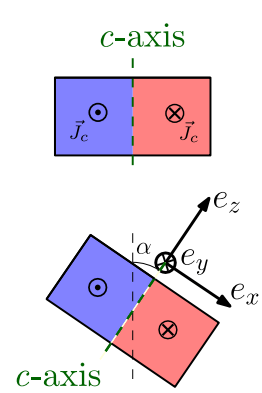

Figure 7. Comparison between the measured trapped field profile in field cooled experiments involving two samples at $65 \mathrm{~K}$ (symbols) and the addition of the modelled contributions coming from each sample (plain lines). (a), (b) and (c): both samples are assumed to be in the critical state, (d) and (e): a cylindrical core free of current is assumed to be present at the centre of the misaligned superconductor.

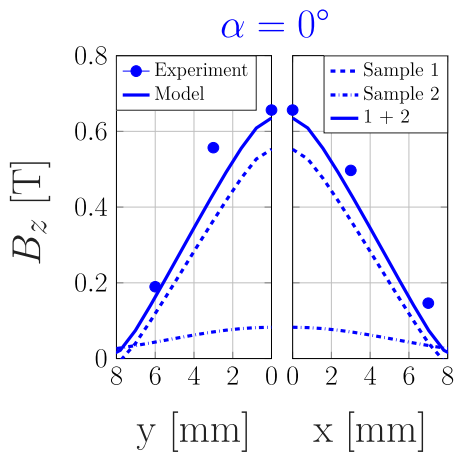

(a)

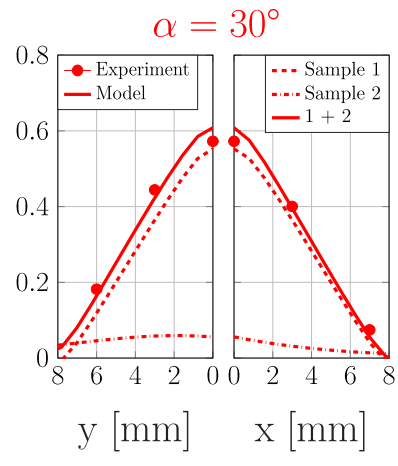

(b)

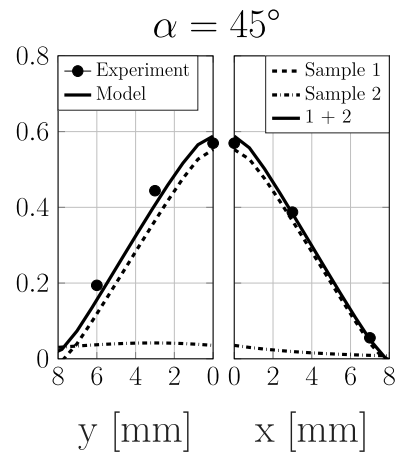

(c)

Figure 8. Comparison between the measured trapped field profile in field cooled experiments involving two samples at $77 \mathrm{~K}$ (symbols) and the addition of the modelled contribution coming from each sample (plain lines). Both samples are assumed to be in the critical state.

ment $45 / 2 / F C$, which is again in agreement with a partial magnetization of the measured sample.

At $77 \mathrm{~K}$, however, the same comparison highlights a beneficial impact of the second sample for all configurations, even for $\alpha=45^{\circ}$. This is again in agreement with the disappearance of the core free of current in the misaligned sample when the temperature of $77 \mathrm{~K}$ is reached.
Comparing, now, the trapped field measurements of experiments $0 / 1 / F C$ and $30 / 1 / F C$, despite the incomplete magnetization of the sample in the misaligned case suggested in the flux creep analysis, no significant difference is observed at $65 \mathrm{~K}$ or at $77 \mathrm{~K}$. Given that in experiment $30 / 2 / F C$ both the flux creep analysis and the trapped field measurements suggest a partial magnetization of the misaligned sample at $65 \mathrm{~K}$, it appears that the aligned sample 
Table 5. Trapped field measured by the central Hall probe at $65 \mathrm{~K}$ and $77 \mathrm{~K}$ on the variable angle sample.

\begin{tabular}{lcc}
\hline Experiment & $B_{c}$ at $65 \mathrm{~K}[\mathrm{~T}]$ & $B_{c}$ at $77 \mathrm{~K}[\mathrm{~T}]$ \\
\hline $0 / 1 / F C$ & 1.1 & 0.55 \\
$30 / 1 / F C$ & 1.09 & 0.53 \\
$30 / 1 / Z F C$ & 1.13 & 0.56 \\
$0 / 2 / F C$ & 1.29 & 0.65 \\
$30 / 2 / F C$ & 1.17 & 0.57 \\
$30 / 2 / Z F C$ & 1.22 & 0.61 \\
$45 / 2 / F C$ & 1.02 & 0.57 \\
\hline
\end{tabular}

has a detrimental effect on the contribution of the misaligned sample to the magnetic flux density.

Focusing on the results of the zero field cooling experiments, it appears that the central value of the trapped field is higher than that measured after a field cooling process in the same configurations. This observation could be attributed to the maximum value of the applied field during the zero field cooling experiments (7 $\mathrm{T})$ which is more than twice higher than that used during the field cooling experiments $(2.5 \mathrm{~T})$. For the misaligned configuration, this leads to a higher penetration of the superconducting current inside the bulk in the zero field cooling experiments which could explain the higher trapped field.

\subsection{Post magnetization approach}

4.2.1. Flux creep analysis. Similarly to the previous experiment, the flux creep measurements were used to fit (5) in order to approximate the critical exponent at the location of each sensor. The critical exponent was again found to lie between 36 and 40 . This gave confidence that the measured sample was completely magnetized.

Equation (5) was also used to evaluate the magnetic flux density at the location of the sensors 20 min after the magnetization of the second sample (beginning of the experiment) and at the end of the last "approach/retract" cycle. The difference between these magnetic flux densities gives an approximation of the drop in trapped field occurring during the "approach/retract" cycles that can be associated to flux creep only. The maximum value of this drop was approximately $6 \mathrm{mT}$. Any smaller drop in the trapped field value can thus hardly be distinguished from flux creep.

4.2.2. Experiments with permanent magnets. First, a translational approach was carried out with permanent magnets, in order to highlight the characteristics that are specific of superconducting trapped field magnets. The evolution of the field measured by the five sensors at the surface of the stationary magnet as a function of the separating distance is presented in figure 9 . In this figure, both the face to face ((a) and (b)) and the sideways approach ((c) and (d)) are represented. An analytical model of the magnetic flux distribution around each magnet was also used to predict the evolution of the measured signals. This model consists in modelling each magnet by an independent coil [45]. The product $n I$, where $n$ is the density of turns and $I$ is the current flowing in the modelled coil, were determined by fitting the expressions in [44] on the magnetic flux profile measured before performing the "approach/retract" cycles. This fitting led to $n I=9.5 \cdot 10^{8} \mathrm{Am}^{-1}$, assumed to be the same for both magnets. For each separation, the addition of the contributions of both coils was computed. The analytical predictions are compared to the measurements in figure 9(b) and (d).

As can be seen from the data presented in figures 9(a) and (c), the magnetic flux density measured by each sensor at the beginning and at the end of the experiments is the same. This means that the "approach/retract" cycles did not cause any irreversible alteration of the magnetization. Such a behaviour is expected since the irreversible demagnetization of Nd-Fe-B magnets usually requires applied field $\mu_{0} H$ exceeding $1 \mathrm{~T}$ [57], which is much larger than the stray fields of the permanent magnets used in the present experiments.

For both kind of approaches, the excellent agreement between calculations and experimental data suggests that the results can be simply understood as the addition of the individual and independent contribution of the flux density generated by each magnet. In the face to face configuration, the decrease of the magnetic flux above one of the magnets due to the second can be unambiguously detected when the separating distance is smaller than approximately 40 $\mathrm{mm}$. From this distance, the measured magnetic flux density monotonically decreases with decreasing separating distance $d$. In the sideways configuration, the decrease of the flux density is apparent when the separating distance is smaller than approximately $20 \mathrm{~mm}$. This decreasing phase corresponds to the addition of the field generated by the stationary magnet with the return field lines of the other. A careful look at figure 9(d) shows that the signals decrease monotonically with decreasing $d$ except for the sensor closest to the movable magnet (number 1). The flux density at this location is found to slightly increase for the last displacement step (dashed zone in figure $9(\mathrm{~d})$ ). This puzzling feature may be understood by considering the evolution of the axial component of the flux density generated by a single permanent magnet above its surface as a function of the radial distance $r$ from the symmetry axis. Starting from a positive magnetic flux density for $r=0$, it decreases for increasing $r$ and even becomes negative for sufficiently large $r$ (because of the return field lines). Considering that the magnetic flux density tends to 0 for $r \rightarrow \infty$, it appears that a minimum should be reached for a finite value of $r$, which explains the minimum observed in the dashed zone in figure $9(\mathrm{~d})$.

4.2.3. Experiments with bulk superconductors. Now we turn to the same experiments carried out with premagnetized superconducting trapped field magnets. Figures 10(a) and (c) shows the flux density acquired by the five sensors as a function of the separating distance. For 
Experimental results
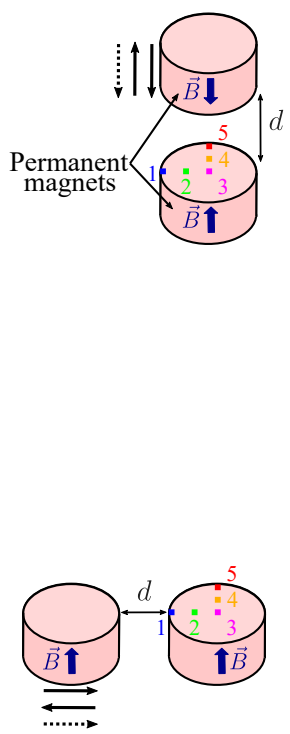

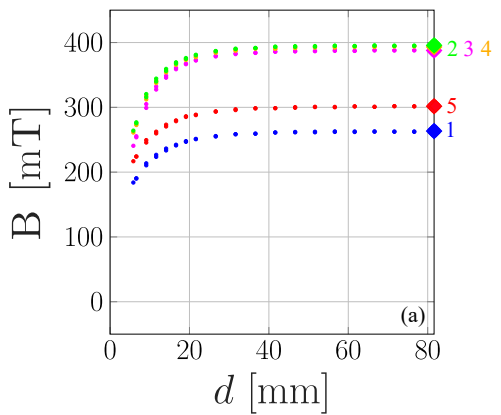

Experimental results

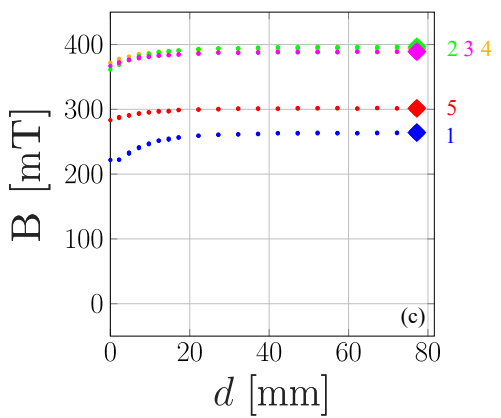

Analytical model

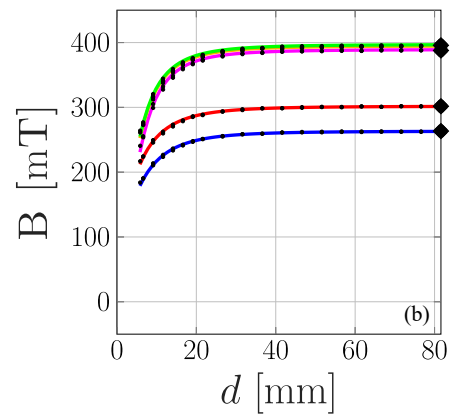

Analytical model

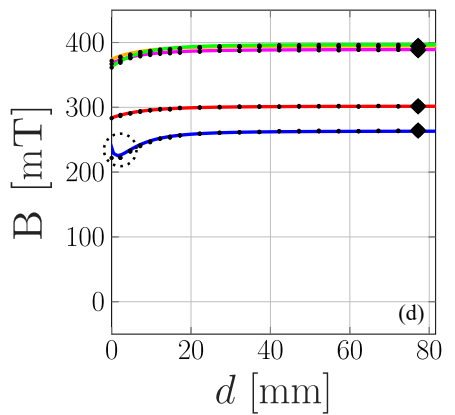

Figure 9. Evolution of the magnetic flux density at the location of the five sensors as a function of the separating distance. Both experimental measurements and analytical calculations are presented. (a) and (b): Face to face approach of permanent magnets (experiment 1), (c) and (d): sideways approach of permanent magnets (experiment 2). The diamond symbols show the starting point of each signal.

each experiment, an enlargement of the signal which is the most affected by the "approach/retract" cycles is also shown in figure $10(\mathrm{~b})$ and $(\mathrm{d})$.

The same general observation as in the case of the permanent magnets can be made here. When the superconducting samples are sufficiently close $(d<20 \mathrm{~mm})$, the contribution of the moving sample at the location of the probes is no longer negligible, which results in a decrease of the measured magnetic flux density. An important difference, however, can be highlighted in comparison to experiments involving permanent magnets: an irreversible drop in the trapped field is observed when comparing the beginning and the end of the experiments.

For a given "approach/retract" cycle in the face to face configuration, the measured signals are found to decrease monotonically for descending values of $d$ (phase 1 , approach) and then to monotonically increase for ascending values of $d$ (phase 2, retract). Focusing on the magnetic flux density measured by the central sensor (figure 10(b)), an irreversible drop in the trapped field is observed when comparing the beginning and the end of each "approach/retract" cycle. The most significant drop happens during the first cycle and had an amplitude of $53 \mathrm{mT}$, i.e. $12.5 \%$ of the initial value. The following drops have amplitudes of $5 \mathrm{mT}, 3 \mathrm{mT}, 2 \mathrm{mT}$ and $2 \mathrm{mT}$ respectively. These amplitudes should be compared to the expected loss of trapped field associated to flux creep which would happen even without performing the "approach/retract" cycles, i.e. $6 \mathrm{mT}$. The first drop is thus definitely not caused by flux creep but is likely to be associated to an irreversible modification of the current distribution in the stationary sample induced by the approaching sample. This modification may be related to the fact that the stray field generated by the approaching sample evaluated on the surface of the stationary one is not strictly parallel to the $c$-axis. Indeed, the flux density contribution of the approaching sample is expected to present a radial component on the surface of the other superconductor in order to keep the magnetic flux density lines closed. To verify this possibility, the analytical model presented in section 3 was used to evaluate an order of magnitude of the magnetic flux density generated by the moving sample on the closest surface of the stationary one. The distance $d$ used in this calculation is equal to $4.4 \mathrm{~mm}$, i.e. the smallest $d$ involved in the experiment. The moduli of the axial and transverse components of the computed magnetic flux density, averaged over the surface of the moving sample, are respectively equal to $85 \mathrm{mT}$ and $50 \mathrm{mT}$. Although the magnetic flux density around the stationary sample is more complex than in a classical crossed field experiment, a demagnetization can be expected for such values of applied field [24,29].

Focusing on the sideways configuration and more particularly on the signal acquired by the closest sensor to the 

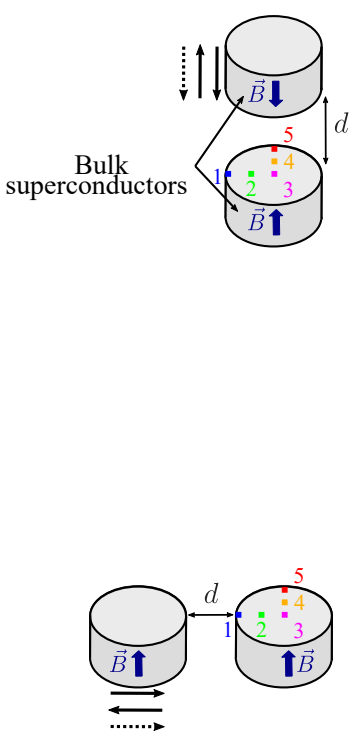
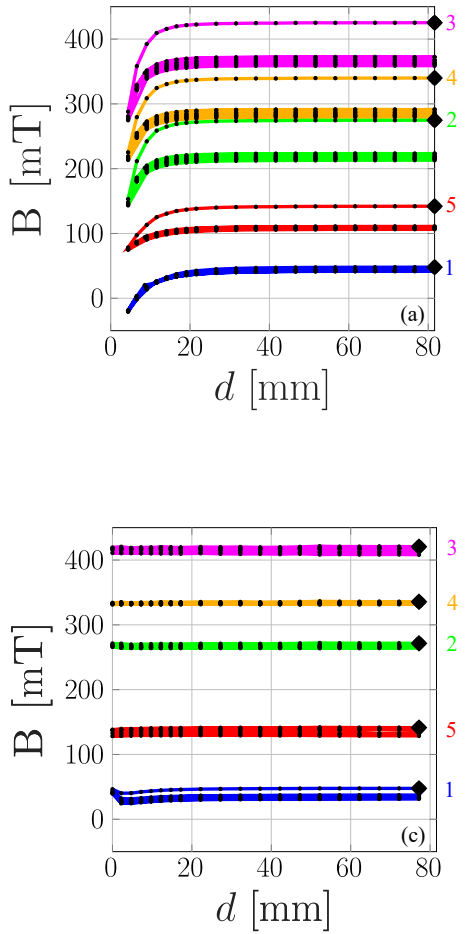
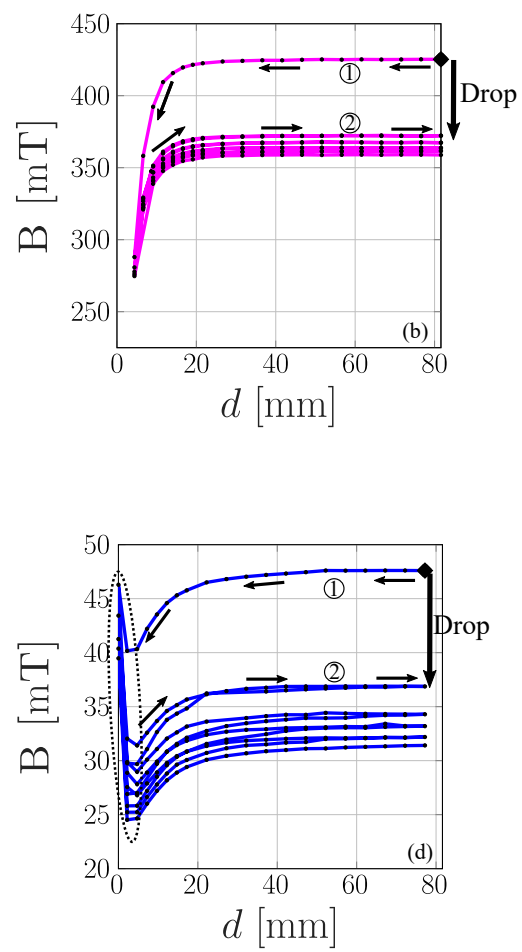

Figure 10. Evolution of the magnetic flux density measured by the five sensors as a function of the separating distance. (a) and (b): Face to face approach of bulk superconductors (experiment 3), (c) and (d): sideways approach of bulk superconductors (experiment 4). The arrows show the path followed during the first "approach/retract" cycle (phase 1: decreasing $d$, phase 2: increasing $d$ ). The diamond symbols show the starting point of each signal.

moving sample (figure 10(d)), a non-monotonic behaviour can be observed. On decreasing $d$, the last two approaching steps are associated to an increase of the flux density at the edge of the sample (dashed zone in figure 10(d)). This non-monotonic behaviour was further investigated by using the analytical model presented in section 3 to try to reproduce the experimental results measured with the considered sensor.

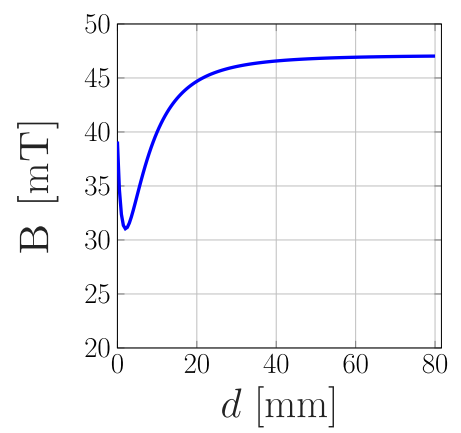

Figure 11. Evolution of the magnetic flux density predicted by the analytical model presented in section 3 at the location of the closest sensor to the moving sample in the sideways configuration, i.e. sensor 1 in figure 10 .

As can be seen from figure 11, the minimum in the evolution of the flux density during the sideways approach of superconductors can be reproduced qualitatively with the analytical model. The non-monotonic behaviour can thus be understood considering a simple addition of the independent contributions of the flux density generated by each superconductor without modification of current density distribution inside them. The feature present in figure $10(d)$ that can not be reproduced by the analytical model, however, is the irreversible drops in trapped field observed at the end of each "approach/retract" cycle which are therefore most likely to be related to a modification of the current distribution in each superconductor. The drop which occurred during the first "approach/retract" cycle has an amplitude of $11 \mathrm{mT}$, i.e. $22.5 \%$ of the initial value. Further drops happened during the remaining cycles, their amplitudes were equal to $3 \mathrm{mT}, 1 \mathrm{mT}, 1 \mathrm{mT}$ and $1 \mathrm{mT}$ respectively. Comparing them to the drop of $6 \mathrm{mT}$ expected from flux creep, the first drop is again most probably caused by the interaction between the superconductors while the following ones are difficult to distinguish from flux creep. Finally, the analytical model presented in section 3 was used to evaluate an order of magnitude of the magnetic flux density generated by the moving sample, assumed to be in the critical state, on the surface of the stationary one for a distance $d$ of $0 \mathrm{~mm}$. The maximum values of the axial and transverse components of the magnetic flux density resulting from these calculations are respectively equal to $300 \mathrm{mT}$ and $260 \mathrm{mT}$. Despite the rather complicated magnetic flux density distribution around the stationary sample, a demagnetization can again be expected for these values of applied field. 


\section{Conclusions}

The flux trapping properties of two $\mathrm{YBa}_{2} \mathrm{Cu}_{3} \mathrm{O}_{7}$ bulk superconductors brought into close proximity was investigated experimentally. The trapped flux density measurements were compared to the analytical calculation of the $\mathbf{B}$-field distribution resulting from the addition of the magnetic field contributions of the two samples. We chose the simplest possible model, in which the samples are assumed to be fully magnetized with persistent currents of constant magnitude $J_{c}$ flowing azimuthally (critical state), even if the sample is tilted with the magnetizing field. We also assume that the current distribution remains unaffected by the presence of the neighbouring sample. Although 3D computer modeling and more elaborated current density distribution could provide a deeper insight of the observed phenomena [49,58-60], the purpose was to show that several experimental facts can be reproduced with a simple model.

It was found that the current distributions in two simultaneously magnetized samples separated by a distance approximately equal to their diameter are not altered by the presence of the neighbouring sample. Although it is likely that the actual current distribution is not purely azimuthal, these results show that when the misorientation angle of the applied field with the $c$-axis is $45^{\circ}$ or less, a simple model assuming that the main flux density component is still along the $c$-axis is able to reproduce the experimental distribution of $\mathbf{B}$. These results agree qualitatively with those obtained for an isolated sample [30,31].

The translational approach of magnetized samples with parallel $c$-axis was also studied at $77 \mathrm{~K}$. Contrary to the behaviour of Nd-Fe-B permanent magnets of similar size and remnant magnetization in the same experimental conditions, the trapped flux density of the superconductors was measured to decay due to the repeated motion cycles. This irreversible decay is most likely associated to a modification of the supercurrent distribution inside the bulk.

The results of both sets of experiments show that despite the close proximity of the samples, it is possible to find configurations in which a significant trapped field is maintained in each superconductor, which is promising for applications involving combined bulk superconductors generating large gradients of the magnetic flux density. More particularly, provided that the $c$-axis component of the magnetizing field is sufficient to magnetize completely each sample, the simultaneous magnetization of two closely spaced bulks led to a situation in which their contributions simply sum thus increasing the magnetic flux density between them. The post-magnetization approach in the sideways configuration led to a much smaller demagnetization effect compared to the face to face approach. Furthermore, only the first "approach/retract" cycle caused a significant decay of the trapped field. Practical applications could thus be designed to be operational with the remaining magnetization after the main decay.

\section{Acknowledgments}

The authors would like to acknowledge the Henry Royce Institute (Equipment grant ref. EP/P024947/1) for financial support. We thank the University of Liege for equipment and travel grants. Michel Houbart is recipient of a FRS-FNRS Research Fellow grant.

\section{References}

[1] JH Durrell, MD Ainslie D Zhou, P Vanderbemden, T Bradshaw, S Speller, M Filipenko, and DA Cardwell 2018 Supercond. Sci. Technol. 31103501

[2] N Saho, N Nishijima, H Tanaka, and A Sasaki 2009 Physica C 469 1286-1289

[3] T Nakamura, D Tamada, Y Yanagi, Y Itoh, T Nemeto, H Utumi, and K Kose 2015 Journal of Magnetic Resonance 259 68-75

[4] MD Ainslie, A George, R Shaw, L Dawson, A Winfield, M Steketee, and S Stockley 2014 Journal of Physics: Conference Series 507032002

[5] D Zhou, M Izumi, M Miki, B Felder, T Ida, and M Kitano 2012 Supercond. Sci. Technol. 25103001

[6] F Fohr, and N Volbers 2018 AIP Advances 8047701

[7] S Nariki, H Teshima, and M Morita 2016 Supercond. Sci. Technol. 29034002

[8] JH Durrell, AR Dennis, J Jaroszynski, MD Ainslie, KGB Palmer, Y-H Shi, AM Campbell, J Hull, M Strasik, EE Hellstrom, and DA Cardwell 2014 Supercond. Sci. Technol. 27082001

[9] M Tomita, M Murakami 2003 Letters to Nature $421517-$ 520

[10] H Fujishiro, T Naito, and S Awaji 2019 Supercond. Sci. Technol. 32045005

[11] H Fujishiro, T Naito, Y Yanagi, Y Itoh, and T Nakamura 2019 Supercond. Sci. Technol. 32065001

[12] FN Werfel, U Floegel-Delor, R Rothfeld, T Riedel, B Goebel, D Wippich, and P Schirrmeister 2012 Supercond. Sci. Technol. 25014007

[13] KS Haran, S Kalsi, T Arndt, H Karmaker, R Badcock, B Buckley, T Haugan, M Izumi, D Loder, JW Bray, P Masson, and EW Stautner 2017 Supercond. Sci. Technol. 30123002

[14] F Mishima, S-I Takeda, Y Izumi, and S Nishijima 2006 IEEE Transactions on Applied Superconductivity 16 367-371

[15] S-I Takeda, F Mishima, S Fujimoto, $\mathrm{Y}$ Izumi, and S Nishijima 2007 Journal of Magnetism and Magnetic Materials 311 367-371

[16] F Mishima, S-I Takeda, Y Izumi, and S Nishijima 2007 IEEE Transactions on Applied Superconductivity $\mathbf{1 7}$ 2303-2306

[17] S Nishijima, S-I Takeda, $F$ Mishima, Y Tabata, M Yamamoto, J-I Joh, H Iseki, Y Muragaki, A Sasaki, and K Jun, N Saho 2008 IEEE Transactions on Applied Superconductivity 18 874-877

[18] SB Kim, I Eritate, T Abe, M Takashashi, S Shima, and A Nakashima 2015 IEEE Transactions on Applied Superconductivity 254602704

[19] T Oka, Y Takahashi, S Yaginuma, J Ogawa, S Fukui, T Sato, K Yokoyama, and T Nakamura 2016 Physics Procedia 81 45-48

[20] LC Barnsley, D Carugo, J Owen, and E Stride 2015 Physics in Medicine and Biology $608303-8327$

[21] K Funaki, and K Yamafuji 1982 Japanese Journal of Applied Physics 21 299-304

[22] AM Campbell, M Baghdadi, A Patel, D Zhou, KY Huang, Y-H Shi, and TA Coombs 2017 Supercond. Sci. Technol. 30034005 
[23] Z Hong, P Vanderbemden, R Pei, Y Jiang, AM Campbell, and TA Coombs 2008 IEEE Transactions on Applied Superconductivity 18 1561-1564

[24] P Vanderbemden, Z Hong, TA Coombs, S Denis, M Ausloos, J Schwartz, IB Rutel, N Hari Babu, DA Cardwell, and AM Campbell 2007 Physical Review B $\mathbf{7 5} 174515$

[25] LM Fisher, AV Kalinov, SE Savel'ev, IF Voloshin, VA Yampol'skii, MAR Leblanc, and S Hirscher 1997 Physica C 278 169-179

[26] J Srpcic, F Perez, KY Huang, Y-H Shi, MD Ainslie, AR Dennis, M Filipenko, M Boll, DA Cardwell, and JH Durell 2019 Supercond. Sci. Technol. 32035010

[27] J Luzuriaga, A Badía-Majós, G Nieva, C López, A Serquis, and G Serrano 2009 Supercond. Sci. Technol. 22015021

[28] M Kapolka, J Srpcic, D Zhou, MD Ainslie, E Pardo, and AR Dennis 2018 IEEE Transaction on Applied Superconductivity $\mathbf{2 8} 6801405$

[29] J Srpcic, F Perez, KY Huang, Y-H Shi, MD Ainslie, AR Dennis, M Filipenko, M Boll, DA Cardwell, and JH Durell 2019 Supercond. Sci. Technol. 32035010

[30] Z Li, T Ida, M Miki, and M Izumi 2017 IEEE Transactions on Applied Superconductivity $\mathbf{2 7} 6800604$

[31] Z Li, T Ida, M Miki, H Teshima, M Morita, and M Izumi 2017 Supercond. Sci. Technol. 30035019

[32] R Kinjo, K Mishima, YW Choi, M Omer, K Yoshida, H Negm, K Torgasin, M Shibata, K Shimahashi, H imon, K Okumura, M Inukai, H Zen, T Kii, K Masuda, K Nagasaki, and H Ohgaki 2014 Physical review special topics - Accelerator and Beams 17022401

[33] T Kii, R Kinjo, N Kimura, M Shibata, MA Bakr, YW Choi, M Omer, K Yoshida, K Ishida, T Komai, K Shimahashi, T Sonobe, H Zen, K Masuda, and H Ohgaki 2012 IEEE Transactions on Applied Superconductivity 224100904

[34] SD Chen, CS Hwang, CM Yang, and IG Chen 2014 IEEE Transaction on Applied Superconductivity 244603005

[35] S Choi, J-H Yoon, B-S Lee, M-S Won, J-W Ok, ZY Zhang, T Kiyoshi, S Matsumoto, S-H Lee 2012 Journal of Applied Physics 111 07E728

[36] K Takahashi, H Fujishiro, MD Ainslie 2018 Supercond. Sci. Technol. 31044005

[37] JR Hull 1999 IEEE Transactions on Applied Superconductivity 9 ASC98

[38] B Shen, J Geng, C Li, X Zhang, L Fu, H Zhang, J Ma, and TA Coombs 2017 Physica C: Superconductivity and its applications $\mathbf{5 3 8} 46-51$

[39] DA Cardwell 1998 Materials Science and Engineering B 53 $1-10$

[40] Y-H Shi, DK Namburi, W Zhao, JH Durrell, AR Dennis, and DA Cardwell 2016 Supercond. Sci. Technol. 29 015010

[41] DK Namburi, Y-H Shi, W Zhai, AR Dennis, JH Durrell, and DA Cardwell 2015 Crystal Growth and Design 15 $1472-1480$

[42] R Bowers 1956 Physical Review 102 1486-1488

[43] National Institute of Standards and Technology Properties of solid materials from cryogenic- to room-temperatures https://trc.nist.gov/cryogenics/materials/material properties.htm

[44] EE Callaghan, and SH Maslen 1960 Nasa Technical Note

[45] IG Chen, J Liu, R Weinstein, and K Lau 1992 Journal of Applied Physics $\mathbf{7 2}$ 1013-1020

[46] CP Bean 1962 Physical Review letters 8 250-253

[47] LM Fisher, AV Kalinov, SE Savel'ev, IF Voloshin, and VA Yampol'skii 1998 Physica C 309 284-294

[48] $\mathrm{Ph}$ Vanderbemden, AD Bradley, RA Doyle, W Lo, DM Astill, DA Cardwell, and AM Campbell 1998 Physica C 302 257-270

[49] M Kapolka, VM Zermeño, S Zou, A Morandi, PL Ribani, E Pardo, and F Grilli 2018 IEEE Transactions on Applied
Superconductivity 288201206

[50] E Zeldov, NM Amer, A Gupta, MW McElfresh, and RJ Gambino 1990 Applied Physics Letters 56 680-682

[51] JZ Sun, CB Eom, B Lairson, JC Bravman, and TH Geballe 1991 Physical Review 43 3002-3008

[52] DA Cardwell, M Murakami, M Zeisberger, W Gawalek, R Gonzalez-Arrabal, M Eisterer, HW Weber, G Fuchs, G Krabbes, A Leenders, HC Freyhardt, X Chaud, R Tournier, and N Hari Babu 2004 Physica C 412-414 623-632

[53] H Yamasaki, and Y Mawatari 2000 Supercond. Sci. Technol. $13202-208$

[54] P Vanderbemden, R Egan, JF Fagnard, B Vanderheyden, M Morita, S Nariki, and H Teshima Presented at the 2016 Applied Superconductivity Conference (ASC), 04/09/2016, Denver, CO, USA Nondestructive measurements of the volume magnetic behavior of large bulk GdBCO single domains and ferromagnet/superconductor structures

[55] EH Brandt 1998 Physical Review B 10 6506-6522

[56] A Sanchez, and C Navau 2001 Physical Review B 64214506

[57] M Katter 2005 Transaction on Magnetics 413853 - 3855

[58] E Pardo, and M Kapolka 2017 Supercond. Sci. Technol. 30 064007

[59] F Grilli, A Morandi, F De Silvestri, and R Brambilla 2018 Supercond. Sci. Technol. 31125003

[60] A Morandi, M Fabbri, PL Ribani, AR Dennis, JH Durrell, Y Shi, and D Cardwell 2018 IEEE Transactions on Applied Superconductivity 283601310 